\title{
Stand-Alone Anterior Cervical Discectomy and Fusion Using an Additive Manufactured Individualized Bioactive Porous Titanium Implant without Bone Graft: Results of a Prospective Clinical Trial
}

\author{
Shunsuke Fujibayashi ${ }^{1}$, Mitsuru Takemoto ${ }^{1}$, Takashi Nakamura ${ }^{1}$, Tomiharu Matsushita ${ }^{2}$, \\ Tadashi Kokubo ${ }^{2}$, Kiyoyuki Sasaki ${ }^{3}$, Shigeo Mori ${ }^{4}$, Shuichi Matsuda ${ }^{1}$ \\ ${ }^{1}$ Department of Orthopedic Surgery, Graduate School of Medicine, Kyoto University, Kyoto, Japan \\ ${ }^{2}$ Department of Biomedical Sciences, College of Life and Health Sciences, Chubu University, Kasugai, Japan \\ ${ }^{3}$ Sagawa Printing Co. Ltd., Muko, Japan \\ ${ }^{4}$ Osaka Yakin Kogyo Co. Ltd., Osaka, Japan
}

The purpose of this study was to introduce our patient-specific bioactive porous titanium implant manufactured using selective laser melting (SLM) and to establish the efficacy and safety of the implant for stand-alone anterior cervical discectomy and fusion (ACDF) based on a prospective clinical trial. We designed a customized ACDF implant using patient-specific data and manufactured the implant using SLM. We produced a bioactive surface through a specific chemical and thermal treatment. Using this implant, we surgically treated four patients with cervical degenerative disc disease and evaluated the clinical and radiological results. We achieved successful bony union in all but one patient without autologous bone grafting within 1 year. We observed no implant subsidence during the follow-up period, and all clinical parameters improved significantly after surgery, with no reported implant-related adverse effects. Our customized bioactive porous titanium implant is a safe and promising implant for stand-alone ACDF.

Keywords: Cervical spine; Anterior cervical discectomy and fusion; Additive manufacturing; Individualized implant; Bioactive porous titanium

\section{Introduction}

Anterior cervical discectomy and fusion (ACDF) with autologous iliac bone graft is the gold standard for treating cervical degenerative disc disease $[1,2]$. However, autologous bone grafts obtained from the anterior iliac crest are associated with several complications, including donor site morbidities, graft collapse, and fusion failure [3-5]. Several types of stand-alone interbody fusion cages have recently been developed; however, cage subsidence and nonunion are still major concerns related to the mismatch of the shape and mechanical properties of cages with ver-

\footnotetext{
Received May 11, 2020; Revised Jun 2, 2020; Accepted Jun 9, 2020

Corresponding author: Shunsuke Fujibayashi

Department of Orthopedic Surgery, Graduate School of Medicine, Kyoto University, Kyoto 606-8507 Japan

Tel: +81-757513366, Fax: +81-757518409, E-mail: shfuji@kuhp.kyoto-u.ac.jp
} 
tebral endplates [6].

Porous titanium and its alloys have the greatest potential as orthopedic implants for load-bearing applications, and their interconnected pore structure can facilitate bone ingrowth. Although there are various methods for producing porous titanium, including particle sintering, spraying a plasma of titanium powder, and compressing and sintering of titanium fibers [7-9], none of these methods can completely control the shape, size, or porosity of the pore.

Rapid prototyping technologies (previously used only for surgical planning) have been demonstrated to be effective rapid manufacturing techniques through additive manufacturing (AM). Selective laser melting (SLM) using titanium powder is an AM technology that can produce porous titanium implants with a particular shape and pore structure [10]. Previous studies have suggested that porous titanium fabricated by AM accelerates osteogenesis [11-13]. The geometric freedom allows for the manufacture of implants with specific structures and mechanical properties, making AM technology especially useful for manufacturing patient-specific devices and implants.

In the current study, we introduce the individualized bioactive porous titanium implant and report the clinical results of this prospective clinical trial.

\section{Technical Note}

\section{Designing methods for the customized implant}

We obtained preoperative computed tomography (CT) scans of the affected cervical spine (1-mm-thick slice) and transferred the CT data into commercially available image processing software (VG Studio; Volume Graphics, Heidelberg, Germany) to reconstruct a three-dimensional (3D) model of the bone structures in a multiplanar reconstruction (MPR) view. The 3D vertebral bone structures were reconstructed by a segmentation process in which the optimal threshold value was selected by visual inspection (Fig. 1). The 3D data of the bone structures were converted to stereolithography (STL) format and transferred to 3D-CAD (computer-aided design) software (FreeForm; SensAble Technologies, Woburn, MA, USA) for implant design. For the basic form of the porous structure, we adapted a diamond crystal lattice, in which each atom is tetrahedron-like and surrounded by four other atoms (Fig. 2). We designed
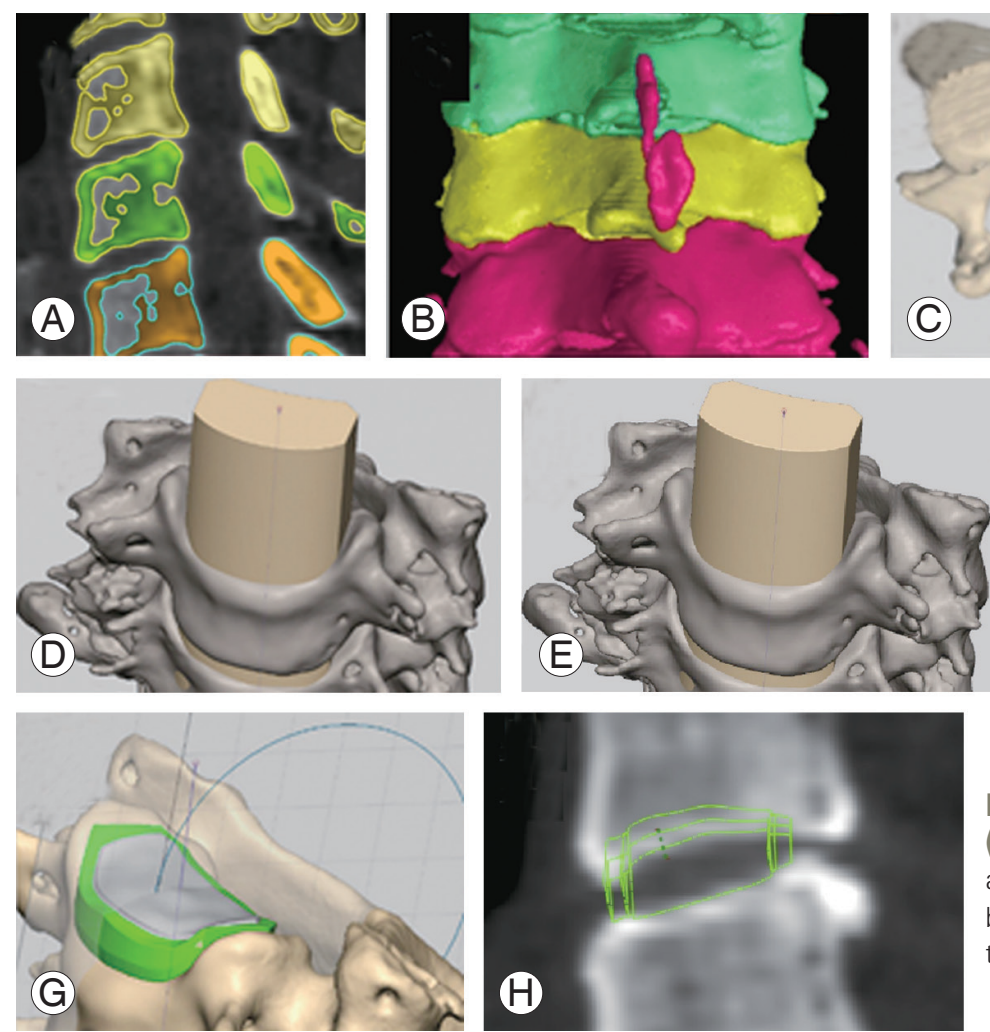

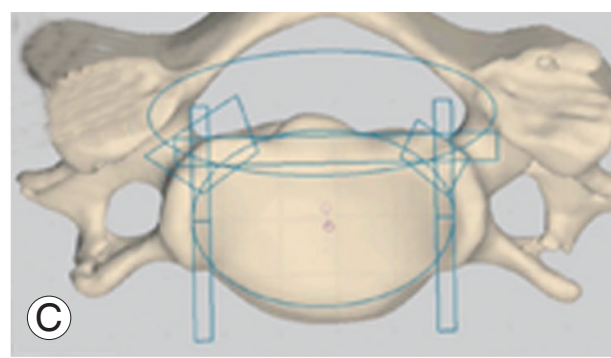

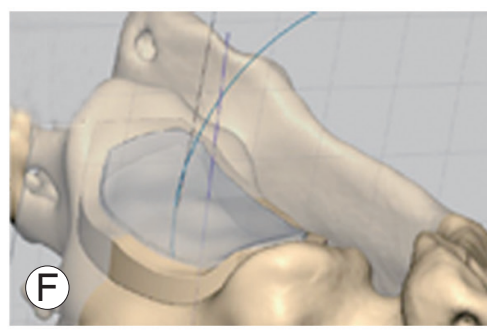

Fig. 1. The design process. (A, B) Segmentation; (C) foot print design; (D, E) basic outline design and building of device; (F) incorporation of porous body; (G) design of size variation; and (H) adaptation on computed tomography image. 

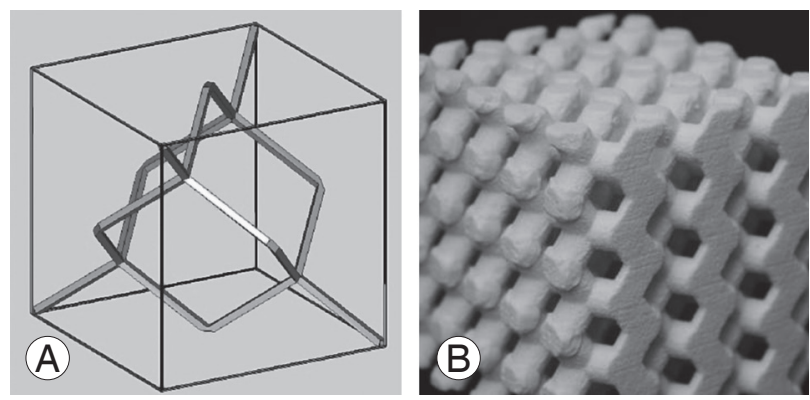

Fig. 2. A single unit (A) and basic structure (B) of diamond crystal lattice.

the manufactured porous titanium implants with a porosity of $65 \%$ and pore sizes of $600 \mu \mathrm{m}$ [14]. The STL format files were transferred to the SLM machine (EOSINT M270; Electro Optical Systems, Munich, Germany).

\section{Fabrication of the individualized implant and bony models}

Individualized titanium implants were fabricated using an SLM method with commercially pure titanium powder (grade 2, particle size $<45 \mu \mathrm{m}$; Osaka Titanium Technologies Co. Ltd., Osaka, Japan). Titanium powder was melted using an ytterbium fiber laser beam in an argon gas atmosphere. The selected slice of the product was then solidified. The top of the previously melted surface was recoated with a $30-\mu \mathrm{m}$-thick layer. Subsequently, selective irradiation was performed using the laser beam. We repeated these steps until the final geometry was achieved. The SLM process parameters were as follows: laser power, $120 \mathrm{~W}$; scanning speed, $200 \mathrm{~mm} / \mathrm{sec}$; hatch spacing, 120 $\mu \mathrm{m}$; and hatch offset, $20 \mu \mathrm{m}$. We manually evaluated the adaptation and stability of the customized implant using the patient-specific bone model (Z-Printer 450; Z Corp., Burlington, MA, USA).

\section{Mechanical properties of the porous titanium body}

We assessed the porous body's mechanical properties in accordance with the International Organization for Standardization (ISO 13314:2011[E]), using porous cylinders (diameter $12 \mathrm{~mm}$, length $12 \mathrm{~mm}$ ) for the mechanical testing. We performed compressive testing using a universal material testing machine (Model EHF-LV020K1-010; Shimadzu Corp., Kyoto, Japan) at a crosshead speed of $1 \mathrm{~mm} /$ min. We calculated the elastic modulus from the slope of the compressive stress-strain curve in the linear elastic
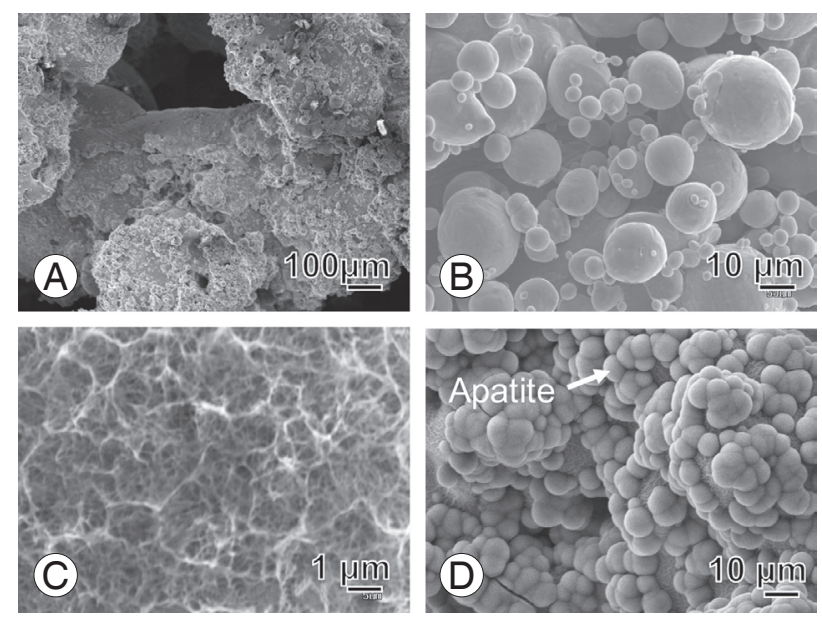

Fig. 3. Apatite formation on selective laser melting porous titanium. (A) Before surface treatment; $(\mathbf{B}, \mathbf{C})$ after surface treatment (B: low magnification view, C: high magnification view); and (D) after immersion in simulated body fluid for 3 days (arrow).

region. We determined the compressive yield strength from the stress-strain curve using the $0.2 \%$ offset method, resulting in an elastic modulus of 648.9 ( \pm standard deviation $[\mathrm{SD}]=9.3) \mathrm{MPa}$ and a compressive yield strength of $42( \pm \mathrm{SD}=1.4) \mathrm{MPa}$.

\section{Bioactive surface treatment}

The customized implants were treated chemically and thermally to provide a bioactive surface, as previously described [15]. Briefly, we immersed the fabricated porous titanium bodies in $5 \mathrm{M}$ aqueous $\mathrm{NaOH}$ solution at $60^{\circ} \mathrm{C}$ for 24 hours, $0.5 \mathrm{mM} \mathrm{HCl}$ at $40^{\circ} \mathrm{C}$ for 24 hours, and ultrapure water at $40^{\circ} \mathrm{C}$ for 24 hours; we then heat treated them at $600^{\circ} \mathrm{C}$ for 1 hour. After the surface treatment, the whole porous surface was uniformly changed to a bioactive thin titanium oxide layer, approximately $1 \mu \mathrm{m}$ thick, with sub-micron-sized pores.

\section{In vitro evaluation of implant bioactivity}

We confirmed the implants' in vitro apatite-forming ability by soaking the samples for 3 days in acellular simulated body fluid (SBF) [16]. The porous body walls were completely covered with apatite within 3 days of soaking in SBF, rendering the whole surface of the implant bioactive with chemical and thermal treatments (Fig. 3). We sterilized the implants by exposing them to $25 \mathrm{kGy} \gamma$ of radiation before their surgical implantation. 


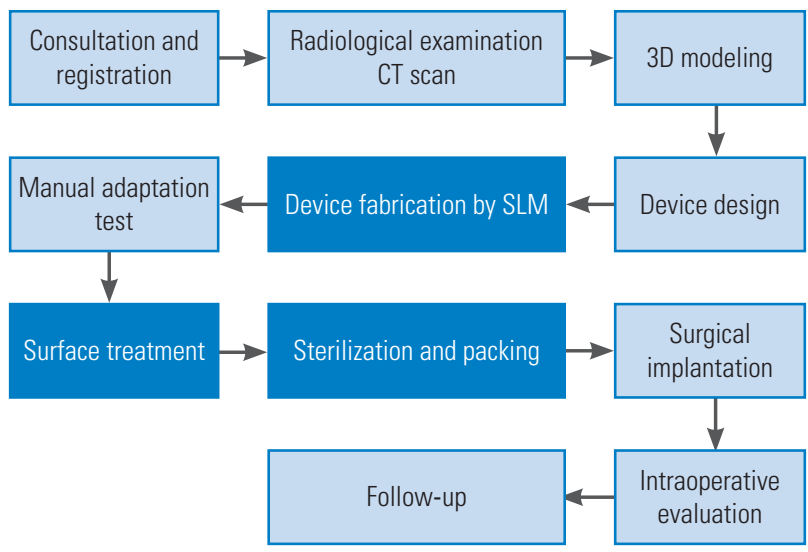

Fig. 4. Process chain of device fabrication and clinical trial. White: in the laboratory; gray: in the hospital; and black: in the factory. CT, computed tomography; 3D, three-dimensional; SLM, selective laser melting.

\section{Patients}

The clinical trial enrolled four patients (three men, one woman; mean age, 62 years; range, $42-75$ years) between January 2013 and October 2013 (Fig. 4). The inclusion criteria were symptomatic single or two-level cervical degenerative disc disease with compression of neural elements, refractory to appropriate conservative treatment for 3 or more months. We included patients with cervical spondylotic myelopathy or disc herniation and excluded those with ossification of the posterior longitudinal ligament. We excluded patients with multilevel (three or more) diseases, previous spinal surgery, history of trauma, general inflammatory disease, or severe comorbidities.

\section{Surgical procedures}

The two senior authors (S.F. and M.T.) performed all of the surgical procedures. Following a standard left anterolateral approach, the authors performed a complete discectomy and neural decompression. The bony endplate was preserved as much as possible to achieve perfect contact of the implant, the latter of which was inserted into the disc space. Stability was confirmed manually after the distractor was removed. Usually, the implants self-locate into the correct position, and moving the cage in any direction is not possible, thereby indicating excellent primary stability. The surgeons chose among three different cage heights in 1-mm steps depending on the intraoperative situation. After the surgery, we instructed the patients to remain in a soft collar for 4 weeks.
Table 1. Summary of preoperative demographic data of patients

\begin{tabular}{lcccccc} 
Case & $\begin{array}{c}\text { Age } \\
\text { (yr) }\end{array}$ & Sex & Diagnosis & Level & $\begin{array}{c}\text { Pre neck } \\
\text { pain VAS }\end{array}$ & $\begin{array}{c}\text { Pre JOA } \\
\text { score }\end{array}$ \\
\hline 1 & 75 & M & CSM & C3/4 & 49 & 10.5 \\
2 & 42 & M & CSM & C5/6 & 78 & 12 \\
\hline 3 & 74 & M & CSM & C4/5 & 46 & 11.5 \\
4 & 57 & F & CSM & C4/5/6 & 6 & 11.5 \\
\hline VAS, Visual Analog Scale; JOA, Japanese Orthopedic Association; M, male; F, \\
female; CSM, cervical spondylotic myelopathy.
\end{tabular}

\section{Radiological assessment}

To assess bony union, we obtained lateral dynamic radiographs at 3,6, and 12 months after the surgery. More than $3^{\circ}$ of motion in flexion-extension was considered nonunion [17]. To evaluate bony union, we assessed coronal and sagittal multidetector $\mathrm{CT}$ reconstruction views at 3 and 12 months. Bone union was defined as complete when there was osseous continuity between the bony endplate and the implant in both the coronal and sagittal multidetector CT images. We defined nonunion as radiolucency $>50 \%$ over the anteroposterior distance of the interface between the endplates and implants. Successful bony union was recorded when the previously mentioned radiological parameter assessments were complete. We calculated cage subsidence from the change in fused segment height (FSH), ascertained using the length of the lines drawn between the center of the cranial endplate of the cranial vertebrae and the center of the caudal end of the caudal vertebrae. We defined significant cage subsidence as an FSH change $\geq 3 \mathrm{~mm}$.

The patients underwent magnetic resonance imaging (MRI) at 1 week and 12 months to assess neural decompression and adverse effects. Three independent experienced spinal surgeons, each with 10 years or more of experience, performed all the radiological assessments. Table 1 summarizes the preoperative clinical and radiological data.

\section{Ethical considerations}

The study was performed in accordance with the principles of the Declaration of Helsinki and the Good Clinical Practices and was registered in the University Hospital Medical Information Network Clinical Trials Registry. In all cases, the patient and his or her relatives were informed 
about the benefits and the risks of the implant. Written informed consent was obtained from all patients and/or their relatives, in accordance with protocols approved by the Institutional Ethics Committee of Kyoto University (C675).

\section{Clinical assessment}

We preoperatively and postoperatively examined the Japanese Orthopaedic Association (JOA) score and its recovery rate (recovery rate=postoperative score-preoperative score/17 [full score]-preoperative score $\times 100 \%$ ). We assessed the self-reported neck pain Visual Analog Scale scores before the operation and at 7 and 14 days after the operation.

\section{Endpoints}

The primary endpoints of this clinical trial were improvement in the clinical results and no implant-related adverse effects. The secondary endpoints were a lack of need for an autologous iliac crest bone graft and bony union with-

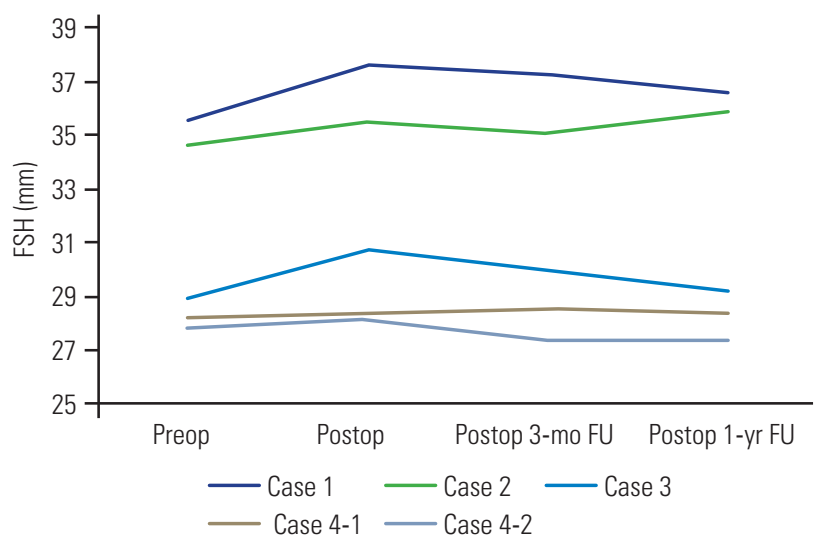

Fig. 5. Serial change of fused segment height. FSH, fused segment height; Preop, preoperative; Postop, postoperative; FU, follow-up. out implant subsidence.

\section{Clinical results}

In all four patients, the preoperative myelopathy and radicular symptoms were resolved immediately after surgery, and the postoperative Visual Analog Scale scores decreased rapidly. There were no surgery-related complications.

\section{Radiological results}

The mean FSH was $31.06 \pm 3.41 \mathrm{~mm}$ preoperatively, $32.06 \pm 3.83 \mathrm{~mm}$ immediately after surgery, $31.7 \pm 3.85 \mathrm{~mm}$ 3 months after surgery, and $31.46 \pm 3.93 \mathrm{~mm} 1$ year after surgery. The FSH values remained steady during the follow-up period, indicating no implant subsidence (Fig. 5). We had complete radiological parameters for all but one patient and considered complete bony union achieved in all but one patient 12 months after surgery. Table 2 summarizes the postoperative clinical and radiological results.

\section{Illustrative case}

A 74-year-old man with progressive myelopathy had a preoperative JOA score of 11.5. Severe spinal cord compression and an intramedullary T2 high intensity zone were revealed at the C4-5 level on MRI. The patient underwent ACDF with an individualized implant without bone graft according to the clinical trial protocol. The patient's postoperative course was uneventful, and his JOA score improved to 16.5 , with a recovery rate of $90.9 \% 1$ year after surgery. The patient achieved radiological bony union within 1 year (Fig. 6).

Table 2. Summary of postoperative demographic data of patients

\begin{tabular}{|c|c|c|c|c|c|c|c|c|c|c|c|}
\hline Case & $\begin{array}{l}\text { Operative } \\
\text { time (min) }\end{array}$ & $\begin{array}{l}\text { Blood loss } \\
(\mathrm{mL})\end{array}$ & $\begin{array}{l}\text { POD7 neck } \\
\text { pain VAS }\end{array}$ & $\begin{array}{l}\text { P0D14 neck } \\
\text { pain VAS }\end{array}$ & $\begin{array}{l}\text { 1-yr JOA } \\
\text { score }\end{array}$ & $\begin{array}{l}\text { 2-yr JOA } \\
\text { score }\end{array}$ & $\begin{array}{l}\text { 5-yr JOA } \\
\text { score }\end{array}$ & $\begin{array}{l}\text { JOA recovery } \\
\text { rate }(\%) \text { at } 5-y r\end{array}$ & ICBG & AEs & Bony union \\
\hline 1 & 119 & 0 & 0 & 0 & 16.5 & 16 & 17 & 100 & - & - & Union \\
\hline 2 & 106 & 0 & 24 & 11 & 14.5 & 16.5 & 16.5 & 90 & - & - & Nonunion \\
\hline 3 & 93 & 0 & 43 & 37 & 16.5 & 16.5 & 16.5 & 90.9 & - & - & Union \\
\hline 4 & 124 & 0 & 3 & 2 & 16.5 & 17 & 17 & 100 & - & - & Union \\
\hline
\end{tabular}

POD, postoperative day; VAS, Visual Analog Scale; JOA, Japanese Orthopedic Association; ICBG, iliac crest bone graft; AEs, adverse effects. 

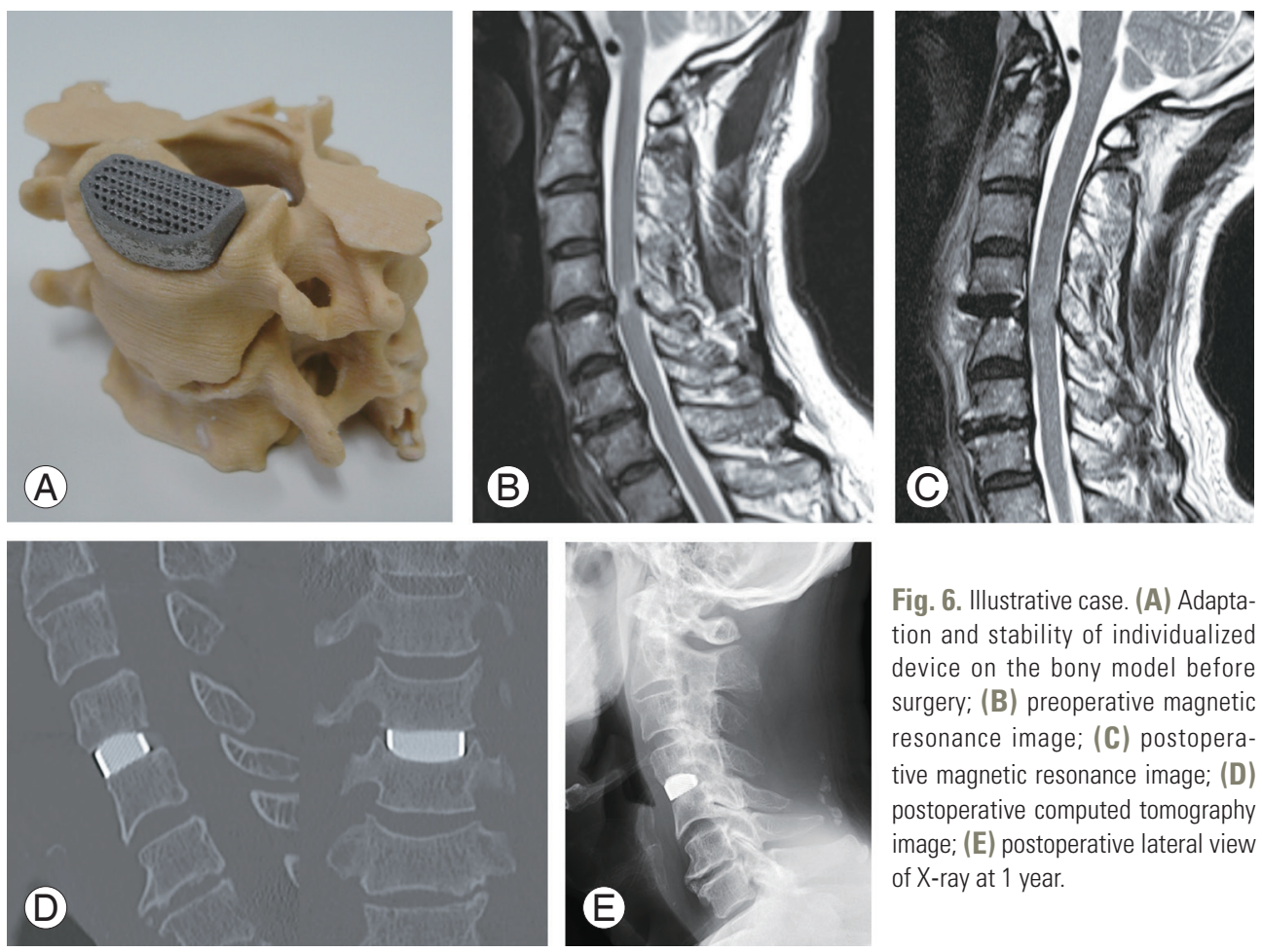

Fig. 6. Illustrative case. (A) Adaptation and stability of individualized device on the bony model before surgery; (B) preoperative magnetic resonance image; (C) postoperative magnetic resonance image; (D) postoperative computed tomography image; (E) postoperative lateral view of $\mathrm{X}$-ray at 1 year.

\section{Discussion}

We successfully treated four patients with degenerative cervical disease using customized bioactive porous titanium implants without bone grafts. The clinical scores improved for all patients, and all but one achieved radiological bony union by the 5-year follow-up.

McGilvray et al. [18] evaluated the bone ingrowth potential of three types of cages in an ovine lumbar fusion model, comparing a polyether ether ketone (PEEK) cage, a titanium-coated PEEK cage, and a 3D-printed porous titanium cage. The results indicated that the 3D-printed porous titanium cage was superior, both biomechanically and histologically [18]. Several case reports have reported successful results following customized implants. Xu et al. [19] reported that Ewing sarcoma of the cervical spine was successfully removed and reconstructed using a customized artificial vertebral body. The implant was manufactured using electron beam technology, and radiological examinations revealed good osseointegration 1 year after surgery [19]. Spetzger et al. [20] reported an ACDF treatment using a customized cervical fusion cage whereby the bearing surface of a commercially-available titanium cage was customized using patient data. Although the authors described the manufacturing process and reported excel- lent primary stability, they did not evaluate the clinical results (such as bony union, cage subsidence, and neurological recovery). Their cage had a similar shape to ours; however, the customization was limited to the bearing surface. Moreover, the surface of other implants was not bioactive. Our implant is not only produced by AM technology using specific patient data but also provides surface bioactivity, providing both mechanical interlocking and biological bonding to the surrounding bone.

When manufacturing customized implants, the most time-consuming process is the design. Although experienced spine surgeons designed the implant for our clinical trial, which reduced the time required, the mean manufacturing period was 2-3 weeks. In the clinical practice, well-trained dedicated engineers should design the implants, and the simulated design should be shown to clinicians. Establishing an efficient processing chain is key to producing customized implants. A manufacturing period of 2-3 weeks might be acceptable for managing degenerative diseases. If we are to apply our technology to trauma surgery, however, reducing the manufacturing period is vital.

This trial had several limitations, the first of which was its small sample size and single-center nature. Second, the application of customized implants was limited to single 
or two-level ACDF. To establish the safety and efficacy of customized implants, further studies are needed to evaluate the application of this technology to other orthopedic fields.

In conclusion, although it is difficult to draw conclusions from this small clinical trial, our individualized bioactive porous titanium implant is a safe and promising implant for stand-alone ACDF, although further studies are needed to improve the design and manufacturing processes.

\section{Conflict of Interest}

No potential conflict of interest relevant to this article was reported.

\section{Funding}

This study was supported by a Grant in Aid for Scientific Research from the Japan Society for the Promotion of Science (no., 19200039).

\section{Author Contributions}

Conception and design: SF, MT, TN, TM, TK, KS, SM; data acquisition: SF, MT, TM, KS, SM; analysis of data: SF, MT, TM, KS, SM; drafting of the manuscript: SF, MT; critical revision: SF, MT, TN, TM, TK, KS, SM; obtaining funding: SF, TN; supervision: TN, TK, SM; and approval of the final manuscript: all authors.

\section{References}

1. Smith GW, Robinson RA. The treatment of certain cervical-spine disorders by anterior removal of the intervertebral disc and interbody fusion. J Bone Joint Surg Am 1958;40-A:607-24.

2. Cloward RB. The anterior approach for removal of ruptured cervical disks. J Neurosurg Spine 2007;6:496511.

3. Banwart JC, Asher MA, Hassanein RS. Iliac crest bone graft harvest donor site morbidity: a statistical evaluation. Spine (Phila Pa 1976) 1995;20:1055-60.

4. Sawin PD, Traynelis VC, Menezes AH. A comparative analysis of fusion rates and donor-site morbidity for autogeneic rib and iliac crest bone grafts in posterior cervical fusions. J Neurosurg 1998;88:255-65.
5. Connolly PJ, Esses SI, Kostuik JP. Anterior cervical fusion: outcome analysis of patients fused with and without anterior cervical plates. J Spinal Disord 1996;9:202-6.

6. Fujibayashi S, Neo M, Nakamura T. Stand-alone interbody cage versus anterior cervical plate for treatment of cervical disc herniation: sequential changes in cage subsidence. J Clin Neurosci 2008;15:1017-22.

7. Fujibayashi S, Neo M, Kim HM, Kokubo T, Nakamura T. Osteoinduction of porous bioactive titanium metal. Biomaterials 2004;25:443-50.

8. Takemoto M, Fujibayashi S, Neo M, Suzuki J, Kokubo T, Nakamura T. Mechanical properties and osteoconductivity of porous bioactive titanium. Biomaterials 2005;26:6014-23.

9. Otsuki B, Takemoto M, Fujibayashi S, Neo M, Kokubo T, Nakamura T. Pore throat size and connectivity determine bone and tissue ingrowth into porous implants: three-dimensional micro-CT based structural analyses of porous bioactive titanium implants. Biomaterials 2006;27:5892-900.

10. Parthasarathy J, Starly B, Raman S, Christensen A. Mechanical evaluation of porous titanium (Ti6Al4V) structures with electron beam melting (EBM). J Mech Behav Biomed Mater 2010;3:249-59.

11. Van Bael S, Chai YC, Truscello S, et al. The effect of pore geometry on the in vitro biological behavior of human periosteum-derived cells seeded on selective laser-melted Ti6Al4V bone scaffolds. Acta Biomater 2012;8:2824-34.

12. Fukuda A, Takemoto M, Saito T, et al. Osteoinduction of porous $\mathrm{Ti}$ implants with a channel structure fabricated by selective laser melting. Acta Biomater 2011;7:2327-36.

13. Pattanayak DK, Fukuda A, Matsushita T, et al. Bioactive Ti metal analogous to human cancellous bone: Fabrication by selective laser melting and chemical treatments. Acta Biomater 2011;7:1398-406.

14. Taniguchi N, Fujibayashi S, Takemoto M, et al. Effect of pore size on bone ingrowth into porous titanium implants fabricated by additive manufacturing: an in vivo experiment. Mater Sci Eng C Mater Biol Appl 2016;59:690-701.

15. Kokubo T, Miyaji F, Kim HM, Nakamura T. Spontaneous formation of bonelike apatite layer on chemically treated titanium metals. J Am Ceram Soc 1996;79:1127-9. 
16. Kokubo T, Kushitani H, Sakka S, Kitsugi T, Yamamuro T. Solutions able to reproduce in vivo surfacestructure changes in bioactive glass-ceramic A-W. J Biomed Mater Res 1990;24:721-34.

17. Kanayama M, Hashimoto T, Shigenobu K, Oha F, Ishida T, Yamane S. Pitfalls of anterior cervical fusion using titanium mesh and local autograft. J Spinal Disord Tech 2003;16:513-8.

18. McGilvray KC, Easley J, Seim HB, et al. Bony ingrowth potential of $3 \mathrm{D}$-printed porous titanium alloy: a direct comparison of interbody cage materi- als in an in vivo ovine lumbar fusion model. Spine J 2018;18:1250-60.

19. Xu N, Wei F, Liu X, et al. Reconstruction of the upper cervical spine using a personalized 3d-printed vertebral body in an adolescent with Ewing sarcoma. Spine (Phila Pa 1976) 2016;41:E50-4.

20. Spetzger U, Frasca M, Konig SA. Surgical planning, manufacturing and implantation of an individualized cervical fusion titanium cage using patient-specific data. Eur Spine J 2016;25:2239-46. 